\title{
Rubber dam use less stressful for children and dentists
}

\author{
Abstracted from \\ Ammann P, Kolb A, Lussi A, Seemann R. \\ Influence of rubber dam on objective and subjective parameters of stress \\ during dental treatment of children and adolescents - a randomized controlled clinical pilot study. \\ Int J Paediatr Dent. 2012 Mar 8 [Epub ahead of print] \\ Address for correspondence R. Seemann, School of Dental Medicine, Freiburgstr. 7, 3010 Bern, \\ Switzerland. Email: rainer.seemann@zmk.unibe.ch
}

\section{Question: When placing fissure sealants does rubber dam compared with cotton wool rolls reduce stress and treatment times?}

Design Randomised controlled trial, single centre and operator. Intervention 72 patients (6-16 years) assessed as compliant, with no allergies, not on significant medication were divided into two groups by 'drawing sealed lots'. 234 fully erupted teeth were sealed. Molars and/ or premolars were sealed dependant on age. Teeth were pre-cleaned with prophy paste. In the controls, teeth were isolated with buccal and lingual cotton rolls and salivary ejector in the intervention group a rubber dam was used. The same etching, rinsing and placement protocol was used in both arms.

Outcome measure Outcomes were: patients' subjective measures of pain using a visual analogue scale; blood pressure (before and after treatment), breath rate, pulse rate and skin resistance at five time points. The operator's pulse rate was measured and they completed a questionnaire on subjective mental and physical stress following treatment. Treatment times were recorded.

Results The breath rate was significantly $(P<0.05)$ lower and the skin resistance level was significantly higher during treatment with rubber dam compared to the control group. Subjective pain perception was significantly lower for the test group. The treatment time needed for the fissure sealing procedure was $12.4 \%$ less in the test group.

Conclusions The authors state; '.. in the hands of an experienced dentist, isolation with rubber dam is less stressful for children and adolescents than isolation with cotton rolls, and can save valuable treatment time.' The operator's stress measures were lower with rubber dam and treatment time was reduced.

\section{Commentary}

Clinical dental research has, at last, started to include patientcentred outcomes, as well as the more usual clinician-centred outcomes, and this paper continues this trend. One of the reasons given for using rubber dam, alongside patient safety and improvement of treatment outcomes and field of vision, is to enhance patient comfort during treatment. The authors report that there is little evidence to support this, and that their study is the first to do so.

The study looked at the stress to operator and patient, of fissure sealant placement under rubber dam, compared with cotton roll isolation, and reported less stress for both with the rubber dam.
Yet few dentists use rubber dam isolation for fissure sealant placement in their child patients, even for restorative work. ${ }^{1}$ The 2009 Cochrane fissure sealant review ${ }^{2}$ included only one study reporting using 'rubber dam if needed', and neither current BSPD $^{3}$ nor $\mathrm{AAPD}^{4}$ guidelines on fissure sealing mention its use in their recommendations. One possible reason is that the optimal time to seal teeth in high caries-risk children is soon after eruption, when placement can be problematic.

So, should this study lead clinicians to start using rubber dam isolation for fissure sealing? As reported, the operator's 'personal preference for rubber dam is certainly a bias for the outcome of this study', giving doubt as to whether it can be considered a 'fair test'. Also, the conclusions don't seem to reflect the uncertainty in the results; although patients reported that subjective pain was lower for both sides of the mouth, none of their physiological outcomes reflected this. Pulse rate, skin resistance and blood pressure were not different between the rubber dam and cotton roll groups at any time points, and where there was a statistically significant difference on one side of the mouth, this was not found for the contralateral side. The only outcome measure in children consistently showing a statistically significant reduction for rubber dam over cotton rolls was their reports of subjective pain perception, but even this finding is complicated by the possibility that these were influenced by operator preferences.

What is most interesting about this paper is that from the operator's physiological measures and self-report he found cotton rolls more stressful to use and the children's results showed that they did not find either technique more stressful. Given the many advantages of rubber dam, this certainly merits consideration.

\section{Nicola Innes}

University of Dundee, Unit of Dental and Oral Health, Park Place, Dundee, Scotland, UK.

1. Soldani, F., \& Foley, J. An assessment of rubber dam usage amongst specialists in paediatric dentistry practising within the UK. Int J Paediatr Dent. 2007; 17: 50-56

2. Ahovuo-Saloranta A, Hiiri A, Nordblad A, Mäkelä M, Worthington HV. Pit and fissure sealants for preventing dental decay in the permanent teeth of children and adolescents. Cochrane Database of Syst Rev. 2008; Issue 4. Art. No. CD001830.

3. Nunn JH, Murray J], Smallridge J; BSPD. British Society of Paediatric Dentistry. British Society of Paediatric Dentistry: a policy document on fissure sealants in paediatric dentistry. Int / Paediatr Dent. 2000; 10: 174-177

4. Beauchamp J, Caufield PW, Crall J], et al. American Dental Association Council on Scientific Affairs. Evidence-based clinical recommendations for the use of pit-andfissure sealants: a report of the American Dental Association Council on Scientific Affairs. J Am Dent Assoc. 2008; 139: 257-268.

Evidence-Based Dentistry (2012) 13, 48. doi:10.1038/sj.ebd.6400859 